\title{
On a symmetry of complex and real multiplication
}

\author{
Igor V. NikOLAEV \\ (Received July 26, 2013; Revised November 13, 2013)
}

\begin{abstract}
It is proved that each lattice with complex multiplication by $f \sqrt{-D}$ corresponds to a pseudo-lattice with real multiplication by $f^{\prime} \sqrt{D}$, where $f^{\prime}$ is an integer defined by $f$.

Key words: complex and real multiplication.
\end{abstract}

\section{Introduction}

The paper continues a study of the duality between elliptic curves with complex multiplication and noncommutative tori with real multiplication initiated in [5]; let us introduce some notation and basic facts. Fix an irrational number $0<\theta<1$; a noncommutative torus is the universal $C^{*}$ algebra $A_{\theta}$ generated by the unitaries $u$ and $v$ satisfying the commutation relation $v u=e^{2 \pi i \theta} u v$ (Rieffel, $1981[6]$ ). Two such tori are stably isomorphic (Morita equivalent) whenever $A_{\theta} \otimes \mathcal{K} \cong A_{\theta^{\prime}} \otimes \mathcal{K}$, where $\mathcal{K}$ is the $C^{*}$-algebra of compact operators; the isomorphism occurs if and only if $\theta^{\prime}=(a \theta+b)$ / $(c \theta+d)$, where $a, b, c, d \in \mathbb{Z}$ and $a d-b c=1$. The K-theory of $A_{\theta}$ is Bott periodic with $K_{0}\left(A_{\theta}\right)=K_{1}\left(A_{\theta}\right) \cong \mathbb{Z}^{2}$. The range of the trace on projections of $A_{\theta} \otimes \mathcal{K}$ is a subset $\Lambda=\mathbb{Z}+\mathbb{Z} \theta$ of the real line (Rieffel, 1981 [6]); $\Lambda$ is called a pseudo-lattice (Manin, 2004 [4]). The torus $A_{\theta}$ is said to have real multiplication if $\theta$ is a quadratic irrationality; we shall denote the set of such algebras by $\mathcal{A}_{R M}$. The real multiplication entails existence of the nontrivial endomorphisms of $\Lambda$ coming from multiplication by the real numbers - hence the name. If $D>1$ is a square-free integer, we shall write $A_{R M}^{(D, f)}$ to denote real multiplication by an order $R_{f}$ of conductor $f \geq 1$ in the field $\mathbb{Q}(\sqrt{D})$; each torus in $\mathcal{A}_{R M}$ can be written in this form (Manin, 2004 [4]).

Let $\mathbb{H}=\{x+i y \in \mathbb{C} \mid y>0\}$ be the upper half-plane and for $\tau \in \mathbb{H}$ let $\mathbb{C} /(\mathbb{Z}+\mathbb{Z} \tau)$ be a complex torus; we routinely identify the latter with a

2000 Mathematics Subject Classification : 11G15 (complex multiplication); 46L85 (noncommutative topology).

Partially supported by NSERC. 
non-singular elliptic curve via the Weierstrass $\wp$ function (Silverman, 1994 $\left[7\right.$, pp.6-7]). Recall that two complex tori are isomorphic, whenever $\tau^{\prime}=$ $(a \tau+b) /(c \tau+d)$, where $a, b, c, d \in \mathbb{Z}$ and $a d-b c=1$. If $\tau$ is an imaginary quadratic number, the elliptic curve is said to have complex multiplication; in this case the lattice $L=\mathbb{Z}+\mathbb{Z} \tau$ admits non-trivial endomorphisms given as multiplication of $L$ by certain complex (quadratic) numbers. Elliptic curves with complex multiplication are fundamental and have long history in number theory; we shall denote the set of such curves by $\mathcal{E}_{C M}$. We write $E_{C M}^{(-D, f)}$ to denote the elliptic curve with complex multiplication by an order $\mathfrak{R}_{f}$ of conductor $f \geq 1$ in the imaginary quadratic field $\mathbb{Q}(\sqrt{-D})$; each curve in $\mathcal{E}_{C M}$ is isomorphic to $E_{C M}^{(-D, f)}$ for some integers $D$ and $f$ (Silverman, 1994 [7, pp. 95-96]).

There exists a covariant functor between elliptic curves and noncommutative tori; the functor maps isomorphic curves to the stably isomorphic tori. To give an idea, let $\phi$ be a closed form on a topological torus; the trajectories of $\phi$ define a measured foliation on the torus. By the HubbardMasur theorem, such a foliation corresponds to a point $\tau \in \mathbb{H}$. The map $F: \mathbb{H} \rightarrow \partial \mathbb{H}$ is defined by the formula $\tau \mapsto \theta=\int_{\gamma_{2}} \phi / \int_{\gamma_{1}} \phi$, where $\gamma_{1}$ and $\gamma_{2}$ are generators of the first homology of the torus. The following is true: (i) $\mathbb{H}=\partial \mathbb{H} \times(0, \infty)$ is a trivial fiber bundle, whose projection map coincides with $F$; (ii) $F$ is a functor, which maps isomorphic complex tori to the stably isomorphic noncommutative tori. We shall refer to $F$ as the Teichmüller functor. It was proved in [5] that $F\left(\mathcal{E}_{C M}\right) \subseteq \mathcal{A}_{R M}$, i.e. $F$ sends elliptic curves with complex multiplication to the noncommutative tori with real multiplication. Namely, $F\left(E_{C M}^{(-D, f)}\right)=A_{R M}^{\left(D, f^{\prime}\right)}$, where $f^{\prime}$ is the least integer satisfying equation $\left|C l\left(R_{f^{\prime}}\right)\right|=\left|C l\left(\Re_{f}\right)\right|$ for the class numbers of orders $R_{f^{\prime}}$ and $\mathfrak{R}_{f}$, respectively; the latter constraint is a necessary and sufficient condition for $A_{R M}^{\left(D, f^{\prime}\right)}$ to discern non-isomorphic curves $E_{C M}^{(-D, f)}$ having the same endomorphism ring $R_{f}$.

Denote by $\Lambda_{R M}^{(D, f)}$ a pseudo-lattice corresponding to the torus $A_{R M}^{(D, f)}$; the $\Lambda_{R M}^{(D, f)}$ can be identified with points of the boundary $\partial \mathbb{H}$ of the half-plane $\mathbb{H}$. Let $x, \bar{x} \in \Lambda_{R M}^{(D, f)}$ be a pair of the conjugate quadratic irrationalities and consider a geodesic half-circle through $x$ and $\bar{x}$ :

$$
\widetilde{\gamma}(x, \bar{x})=\frac{x e^{t / 2}+i \bar{x} e^{-t / 2}}{e^{t / 2}+i e^{-t / 2}}, \quad-\infty \leq t \leq \infty .
$$


A Riemann surface $X$ is said to be associated to $A_{R M}^{(D, f)}$, if the covering of the geodesic spectrum of $X$ contains the set $\left\{\widetilde{\gamma}(x, \bar{x}): \forall x \in \Lambda_{R M}^{(D, f)}\right\}$, see Definition 1; such a surface will be denoted by $X\left(A_{R M}^{(D, f)}\right)$. Our main result can be expressed as follows.

Theorem 1 For every square-free integer $D>1$ and integer $f \geq 1$ there exists a holomorphic map $F^{-1}: X\left(A_{R M}^{\left(D, f^{\prime}\right)}\right) \rightarrow E_{C M}^{(-D, f)}$, where $F\left(E_{C M}^{(-D, f)}\right)=A_{R M}^{\left(D, f^{\prime}\right)}$.

The note is organized as follows. Section 2 is reserved for notation and preliminary facts. Theorem 1 is proved in Section 3.

\section{Riemann surface $X\left(A_{R M}^{(D, f)}\right)$}

Let $X$ be a Riemann surface; consider the geodesic spectrum of $X$, i.e. the set Spec $X$ consisting of all closed geodesics of $X$. Recall that for the covering map $\mathbb{H} \rightarrow X$ each geodesic $\gamma \in \operatorname{Spec} X$ is the image of a geodesic half-circle $\widetilde{\gamma}\left(x, x^{\prime}\right) \in \mathbb{H}$ with the endpoints $x \neq x^{\prime}$. Denote by $\widetilde{\operatorname{spec}} X \subset \mathbb{H}$ the set of geodesic half-circles covering the geodesic spectrum of $X$.

Definition 1 We shall say that the Riemann surface $X$ is associated to the noncommutative torus $A_{R M}^{(D, f)}$, if $\left\{\widetilde{\gamma}(x, \bar{x}): \forall x \in \Lambda_{R M}^{(D, f)}\right\} \subset \widetilde{\operatorname{Spec}} X$; the associated Riemann surface will be denoted by $X\left(A_{R M}^{(D, f)}\right)$.

Let $N \geq 1$ be an integer; by $\Gamma_{1}(N)$ we understand a subgroup of the modular group $S L_{2}(\mathbb{Z})$ consisting of matrices of the form

$$
\left\{\left(\begin{array}{ll}
a & b \\
c & d
\end{array}\right) \in S L_{2}(\mathbb{Z}) \mid a, d \equiv 1 \bmod N, c \equiv 0 \bmod N\right\} ;
$$

the corresponding Riemann surface $\mathbb{H} / \Gamma_{1}(N)$ will be denoted by $X_{1}(N)$. The following lemma links $X\left(A_{R M}^{(D, f)}\right)$ to $X_{1}(N)$.

Lemma $1 \quad X\left(A_{R M}^{(D, f)}\right) \cong X_{1}(f D)$.

Proof. Let $\Lambda_{R M}^{(D, f)}$ be a pseudo-lattice with real multiplication by an order $R$ in the real quadratic number field $\mathbb{Q}(\sqrt{D})$; it is known, that $\Lambda_{R M}^{(D, f)} \subseteq R$ and $R=\mathbb{Z}+(f \omega) \mathbb{Z}$, where $f \geq 1$ is the conductor of $R$ and 


$$
\omega= \begin{cases}\frac{1+\sqrt{D}}{2} & \text { if } D \equiv 1 \bmod 4 \\ \sqrt{D} & \text { if } D \equiv 2,3 \bmod 4\end{cases}
$$

see e.g. (Borevich \& Shafarevich, 1988 [1, pp. 130-131]) Recall that matrix $(a, b, c, d) \in S L_{2}(\mathbb{Z})$ has a pair of real fixed points $x$ and $\bar{x}$ if and only if $|a+d|>2$ (the hyperbolic matrix); the fixed points can be found from the equation $x=(a x+b)(c x+d)^{-1}$ by the formulas:

$$
x=\frac{a-d}{2 c}+\sqrt{\frac{(a+d)^{2}-4}{4 c^{2}}}, \quad \bar{x}=\frac{a-d}{2 c}-\sqrt{\frac{(a+d)^{2}-4}{4 c^{2}}} .
$$

Case I. If $D \equiv 1 \bmod 4$, then formula (3) implies that $R=(1+f / 2) \mathbb{Z}+$ $\left(\sqrt{f^{2} D} / 2\right) \mathbb{Z}$. If $x \in \Lambda_{R M}^{(D, f)}$ is fixed point of a transformation $(a, b, c, d) \in$ $S L_{2}(\mathbb{Z})$, then formula (4) implies:

$$
\left\{\begin{aligned}
\frac{a-d}{2 c} & =\left(1+\frac{f}{2}\right) z_{1} \\
\frac{(a+d)^{2}-4}{4 c^{2}} & =\frac{f^{2} D}{4} z_{2}^{2}
\end{aligned}\right.
$$

for some integer numbers $z_{1}$ and $z_{2}$. The second equation can be written in the form $(a+d)^{2}-4=c^{2} f^{2} D z_{2}^{2} ;$ we have therefore $(a+d)^{2} \equiv 4 \bmod (f D)$ and $a+d \equiv \pm 2 \bmod (f D)$. Without loss of generality we assume $a+d \equiv$ $2 \bmod (f D)$ since matrix $(a, b, c, d) \in S L_{2}(\mathbb{Z})$ can be multiplied by -1 . Notice that the last equation admits a solution $a=d \equiv 1 \bmod (f D)$.

The first equation yields us $(a-d) / c=(2+f) z_{1}$, where $c \neq 0$ since the matrix $(a, b, c, d)$ is hyperbolic. Notice that $a-d \equiv 0 \bmod (f D)$; since the ratio $(a-d) / c$ must be integer, we conclude that $c \equiv 0 \bmod (f D)$. All together, we get:

$$
a \equiv 1 \bmod (f D), \quad d \equiv 1 \bmod (f D), \quad c \equiv 0 \bmod (f D) .
$$

Case II. If $D \equiv 2$ or $3 \bmod 4$, then $R=\mathbb{Z}+\left(\sqrt{f^{2} D}\right) \mathbb{Z}$. If $x \in \Lambda_{R M}^{(D, f)}$ is fixed point of a transformation $(a, b, c, d) \in S L_{2}(\mathbb{Z})$, then formula (4) implies: 


$$
\left\{\begin{aligned}
\frac{a-d}{2 c} & =z_{1} \\
\frac{(a+d)^{2}-4}{4 c^{2}} & =f^{2} D z_{2}^{2}
\end{aligned}\right.
$$

for some integer numbers $z_{1}$ and $z_{2}$. The second equation gives $(a+d)^{2}-$ $4=4 c^{2} f^{2} D z_{2}^{2}$; therefore $(a+d)^{2} \equiv 4 \bmod (f D)$ and $a+d \equiv \pm 2 \bmod (f D)$. Again without loss of generality we assume $a+d \equiv 2 \bmod (f D)$ since matrix $(a, b, c, d) \in S L_{2}(\mathbb{Z})$ can be multiplied by -1 . The last equation admits a solution $a=d \equiv 1 \bmod (f D)$.

The first equation is $(a-d) / c=2 z_{1}$, where $c \neq 0$. Since $a-d \equiv$ $0 \bmod (f D)$ and the ratio $(a-d) / c$ must be integer, one concludes that $c \equiv$ $0 \bmod (f D)$. All together, we get equations (6). Since all possible cases are exhausted, Lemma 1 follows.

Remark 1 There exist other finite index subgroups of $S L_{2}(\mathbb{Z})$ whose geodesic spectrum contains the set $\left\{\widetilde{\gamma}(x, \bar{x}): \forall x \in \Lambda_{R M}^{(D, f)}\right\}$; however $\Gamma_{1}(f D)$ is a unique group with such a property among subgroups of the principal congruence group.

Remark 2 Not all geodesics of $X_{1}(f D)$ have form (1); thus the set $\left\{\widetilde{\gamma}(x, \bar{x}): \forall x \in \Lambda_{R M}^{(D, f)}\right\}$ is strictly included in the geodesic spectrum of modular curve $X_{1}(f D)$.

\section{Proof of Theorem 1}

Recall, that $\Gamma(N):=\left\{(a, b, c, d) \in S L_{2}(\mathbb{Z}) \mid a, d \equiv 1 \bmod N, b, c \equiv\right.$ $0 \bmod N\}$ is called a principal congruence group of level $N$; the corresponding (compact) Riemann surface will be denoted by $X(N)=\mathbb{H} / \Gamma(N)$.

Lemma 2 (Hecke) There exists a holomorphic map $X(f D) \rightarrow E_{C M}^{(-D, f)}$.

Proof. A detailed proof of this beautiful fact is given in (Hecke, 1928 [3]).

To give an idea of the proof, let $\mathfrak{R}$ be an order of conductor $f \geq 1$ in the imaginary quadratic number field $\mathbb{Q}(\sqrt{-D})$; consider an $L$-function attached to $\mathfrak{R}$ :

$$
L(s, \psi)=\prod_{\mathfrak{P} \subset \mathfrak{R}} \frac{1}{1-\psi(\mathfrak{P}) / N(\mathfrak{P})^{s}}, \quad s \in \mathbb{C},
$$


where $\mathfrak{P}$ is a prime ideal in $\mathfrak{R}, N(\mathfrak{P})$ its norm and $\psi$ a Grössencharacter. A crucial observation (Section 1 ) says that the series $L(s, \psi)$ converges to a cusp form $w(s)$ of the principal congruence group $\Gamma(f D)$.

By the Deuring Theorem, $L\left(E_{C M}^{(-D, f)}, s\right)=L(s, \psi) L(s, \bar{\psi})$, where $L\left(E_{C M}^{(-D, f)}, s\right)$ is the Hasse-Weil $L$-function of the elliptic curve and $\bar{\psi}$ a conjugate of the Grössencharacter, see (Silverman, 1994 [7, p. 175]); moreover $L\left(E_{C M}^{(-D, f)}, s\right)=L(w, s)$, where $L(w, s):=\sum_{n=1}^{\infty} \frac{c_{n}}{n^{s}}$ and $c_{n}$ the Fourier coefficients of the cusp form $w(s)$. In other words, $E_{C M}^{(-D, f)}$ is a modular elliptic curve.

One can now apply the modularity principle: if $A_{w}$ is an abelian variety given by the periods of holomorphic differential $w(s) d s$ (and its conjugates) on $X(f D)$, then the following diagram commutes

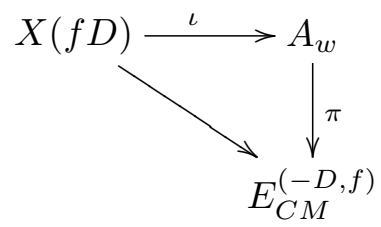

The holomorphic map $X(f D) \rightarrow E_{C M}^{(-D, f)}$ is obtained as a composition of the canonical embedding $\iota: X(f D) \rightarrow A_{w}$ with the subsequent holomorphic projection $\pi: A_{w} \rightarrow E_{C M}^{(-D, f)}$.

Lemma 3 The functor $F$ acts by the formula $E_{C M}^{(-D, f)} \mapsto A_{R M}^{\left(D, f^{\prime}\right)}$.

Proof. Let $L_{C M}$ be a lattice with complex multiplication by an order $\mathfrak{R}=$ $\mathbb{Z}+(f \omega) \mathbb{Z}$ in the imaginary quadatic field $\mathbb{Q}(\sqrt{-D})$; the multiplication by $\alpha \in \Re$ generates an endomorphism $(a, b, c, d) \in M_{2}(\mathbb{Z})$ of the lattice $L_{C M}$. It is known, that the endomorphisms of lattice $L_{C M}$ and endomorphisms of the pseudo-lattice $\Lambda_{R M}=F\left(L_{C M}\right)$ are related by the following explicit $\operatorname{map}[4$, p. 524]:

$$
\left(\begin{array}{ll}
a & b \\
c & d
\end{array}\right) \in \operatorname{End}\left(L_{C M}\right) \longmapsto\left(\begin{array}{cc}
a & b \\
-c & -d
\end{array}\right) \in \operatorname{End}\left(\Lambda_{R M}\right) .
$$

Moreover, one can always assume $d=0$ in a proper basis of $L_{C M}$. We shall consider the following two cases. 
Case I. If $D \equiv 1 \bmod 4$ then by (3) $\mathfrak{R}=\mathbb{Z}+\left(\left(f+\sqrt{-f^{2} D}\right) / 2\right) \mathbb{Z}$; thus the multiplier $\alpha=(2 m+f n) / 2+\sqrt{\left(-f^{2} D n^{2}\right) / 4}$ for some $m, n \in \mathbb{Z}$. Therefore multiplication by $\alpha$ corresponds to an endomorphism $(a, b, c, 0) \in$ $M_{2}(\mathbb{Z})$, where

$$
\left\{\begin{array}{l}
a=\operatorname{Tr}(\alpha)=\alpha+\bar{\alpha}=2 m+f n \\
b=-1 \\
c=N(\alpha)=\alpha \bar{\alpha}=\left(\frac{2 m+f n}{2}\right)^{2}+\frac{f^{2} D n^{2}}{4} .
\end{array}\right.
$$

To calculate a primitive generator of endomorphisms of the lattice $L_{C M}$ one should find a multiplier $\alpha_{0} \neq 0$ such that

$$
\left|\alpha_{0}\right|=\min _{m . n \in \mathbb{Z}}|\alpha|=\min _{m . n \in \mathbb{Z}} \sqrt{N(\alpha)} .
$$

From the last equation of (10) the minimum is attained for $m=-f / 2$ and $n=1$ if $f$ is even or $m=-f$ and $n=2$ if $f$ is odd. Thus

$$
\alpha_{0}= \begin{cases} \pm \frac{f}{2} \sqrt{-D}, & \text { if } f \text { is even } \\ \pm f \sqrt{-D}, & \text { if } f \text { is odd }\end{cases}
$$

To find the matrix form of the endomorphism $\alpha_{0}$, we shall substitute in (9) $a=d=0, b=-1$ and $c=f^{2} D / 4$ if $f$ is even or $c=f^{2} D$ if $f$ is odd. Thus the Teichmüller functor maps the multiplier $\alpha_{0}$ into

$$
F\left(\alpha_{0}\right)= \begin{cases} \pm \frac{f^{\prime}}{2} \sqrt{D}, & \text { if } f^{\prime} \text { is even } \\ \pm f^{\prime} \sqrt{D}, & \text { if } f^{\prime} \text { is odd }\end{cases}
$$

Comparing equations (12) and (13) one verifies that formula $F\left(E_{C M}^{(-D, f)}\right)=$ $A_{R M}^{\left(D, f^{\prime}\right)}$ is true in this case.

Case II. If $D \equiv 2$ or $3 \bmod 4$ then by $(3) \mathfrak{R}=\mathbb{Z}+\left(\sqrt{-f^{2} D}\right) \mathbb{Z}$; thus the multiplier $\alpha=m+\sqrt{-f^{2} D n^{2}}$ for some $m, n \in \mathbb{Z}$. A multiplication by $\alpha$ corresponds to an endomorphism $(a, b, c, 0) \in M_{2}(\mathbb{Z})$, where 


$$
\left\{\begin{array}{l}
a=\operatorname{Tr}(\alpha)=\alpha+\bar{\alpha}=2 m \\
b=-1 \\
c=N(\alpha)=\alpha \bar{\alpha}=m^{2}+f^{2} D n^{2}
\end{array}\right.
$$

We shall repeat the argument of Case I; then from the last equation of (14) the minimum of $|\alpha|$ is attained for $m=0$ and $n= \pm 1$. Thus $\alpha_{0}= \pm f \sqrt{-D}$.

To find the matrix form of the endomorphism $\alpha_{0}$ we substitute in (9) $a=d=0, b=-1$ and $c=f^{2} D$. Thus the Teichmüller functor maps the multiplier $\alpha_{0}= \pm f \sqrt{-D}$ into $F\left(\alpha_{0}\right)= \pm f^{\prime} \sqrt{D}$. In other words, formula $F\left(E_{C M}^{(-D, f)}\right)=A_{R M}^{\left(D, f^{\prime}\right)}$ is true in this case as well.

Since all possible cases are exhausted, Lemma 3 is proved.

Lemma 4 For every $N \geq 1$ there exists a holomorphic map $X_{1}(N) \rightarrow$ $X(N)$.

Proof. Indeed, $\Gamma(N)$ is a normal subgroup of index $N$ of the group $\Gamma_{1}(N)$; therefore there exists a degree $N$ holomorphic map $X_{1}(N) \rightarrow X(N)$.

Theorem 1 follows from Lemmas $1-3$ and Lemma 4 for $N=f D$.

Remark 3 While this note was in print, the author came across a preprint (D'Andrea, Fiore \& Franco, 2013 [2]). Using the idea of quantum deformation of the line bundles over elliptic curves, the authors establish a remarkable formula

$$
\tau-\frac{p \theta}{2} i \in \mathbb{Z}+\mathbb{Z} i
$$

where $p \in \mathbb{Z}$ is the first Chern class of the line bundle. The reader is encouraged to verify, that Theorem 1 satisfies equation (15) for a line bundle of the Chern class $p=2 f^{\prime}$ with $\tau=f \sqrt{-D}$ and $\theta=\sqrt{D}$.

Acknowledgment I thank the referee for helpful comments.

\section{References}

[ 1 ] Borevich Z. I. and Shafarevich I. R., Number Theory, Acad. Press, 1966.

[ 2 ] D'Andrea F., Fiore G. and Franco D., Modules over the noncommutative torus and elliptic curves. Lett. Math. Phys. 104 (2014), 1425-1443. 
[ 3 ] Hecke E., Bestimmung der Perioden gewisser Integrale durch die Theorie der Klassenkörper. Math. Z. 28 (1928), 708-727.

[ 4 ] Manin Yu. I., Real multiplication and noncommutative geometry, in "Legacy of Niels Hendrik Abel", 685-727, Springer, 2004.

[5] Nikolaev I., Remark on the rank of elliptic curves. Osaka J. Math. 46 (2009), 515-527.

[6 ] Rieffel M. A., $C^{*}$-algebras associated with irrational rotations. Pacific J. of Math. 93 (1981), 415-429.

[ 7 ] Silverman J. H., Advanced Topics in the Arithmetic of Elliptic Curves. GTM 151, Springer 1994.

The Fields Institute for Research in Mathematical Sciences Toronto, ON, Canada

E-mail: igor.v.nikolaev@gmail.com

Current address:

1505-657 Worcester St., Southbridge, MA 01550, U.S.A. 